\title{
Explaining Patients' Beliefs About the Neces- sity and Harmfulness of Antidepressants
}

\author{
James E. Aikens, $P b D^{1,2}$ \\ Donald E. Nease, Jr, MD \\ Michael S. Klinkman, $M D, M S^{1,2}$ \\ 'Department of Family Medicine, \\ University of Michigan Medical School, \\ Ann Arbor \\ ${ }^{2}$ Department of Psychiatry, University of \\ Michigan Medical School, Ann Arbor
}

\begin{abstract}
PURPOSE Patients' beliefs about antidepressants vary widely and probably influence adherence, yet little is known about what underlies such beliefs. This study's objective was to identify the demographic and clinical characteristics that account for patients' beliefs about antidepressants.
\end{abstract}

METHODS Participants were 165 patients with unipolar nonpsychotic major depression from primary care and psychiatry clinics who were participating in the baseline phase of a multistaged trial of medication and psychotherapy. Before patients started antidepressants, interview and self-report measures were used to assess treatment beliefs, depression features, and comorbid conditions. Linear multivariate regression was used to identify the strongest correlates of perceived medication necessity and harmfulness after adjusting for age, sex, education, and the random effects of patients within clinical site.

RESULTS Perceived necessity was associated with older age $(P<.001)$, more severe symptoms $(P=.03)$, longer anticipated duration of symptoms $(P=.001)$, and attribution of symptoms to chemical imbalance $(P=.005)$. Perceived harmfulness was highest among patients who had not taken antidepressants before $(P=.02)$, attributed their symptoms to random factors $(P=.04)$, and had a subjectively unclear understanding of depression $(P=.003)$. Neither belief was significantly associated with sex, education, age at first depressive episode, presence of melancholia or anxiety, psychiatric comorbidity, or clinical setting.

CONCLUSIONS Skepticism about antidepressants is strongest among younger patients who have never taken antidepressants, view their symptoms as mild and transient, and feel unclear about the factors affecting their depression. Perhaps these patients would benefit the most from adherence promotion focusing on treatment beliefs.

Ann Fam Med 2008;6:23-29. DOI: 10.1370/afm.759.

\section{INTRODUCTION}

$\Lambda$ lthough depression treatment guidelines recommend continuation of medication for at least 8 months after symptom remission, ${ }^{1-3}$ $50 \%$ to $83 \%$ of patients prescribed antidepressants either discontinue their medication prematurely or take it too inconsistently to derive any clinical benefit ${ }^{4-6}$ which appears to increase their risks for relapse and recurrence. ${ }^{7-9}$ A large body of literature indicates that patients' beliefs and attitudes about medication predict medication adherence, treatment outcome, or both - in general ${ }^{10}$ and in depression. ${ }^{4,5,11-13}$ Our prior study of 573 primary care patients indicated that the only identifiable baseline predictor of early discontinuation was beliefs about the appropriateness of taking medication for depression. ${ }^{11}$ In another study of patients who were well established in the maintenance phase of antidepressant therapy, we found patient beliefs about antidepressant necessity vs harmfulness to be the only identifiable correlate of adherence. ${ }^{14}$ In neither study did demographic or depression characteristics account for adherence.

Despite this growing literature, little is known about what underlies 
antidepressant beliefs. Because studies to promote antidepressant adherence have yielded disappointing findings, ${ }^{15-19}$ a better understanding of treatment beliefs may help improve the patient-centeredness of depression care and suggest innovative new intervention strategies. We focused on patients' beliefs about the necessity and harmfulness of antidepressants because of prior evidence of their robust linkage to adherence in both psychiatric and nonpsychiatric conditions. ${ }^{20-23}$ If perceptions of necessity and harmfulness have different correlates, moreover, then different clinical responses might be indicated for each.

The study's primary objective was to identify the demographic and clinical characteristics that account for patients' beliefs about antidepressants. A secondary objective was to explore the role of patients' cognitive representations of their depressive symptoms. According to Leventhal's Illness Representations Model, ${ }^{24}$ patients construe their symptoms as having a certain label, cause, expected timeline, consequences, and controllability. Although it is unclear whether symptom representations influence antidepressant adherence, one preliminary study suggested higher levels of nonadherence among patients who attribute their depression to interpersonal causes, ${ }^{25}$ whereas another study paradoxically showed the best clinical outcomes among patients who view their depressive symptoms in nonbiological terms ${ }^{26}$ Given these inconsistencies, we sought to clarify the role of depression representations

\section{METHODS}

\section{Design}

The cross-sectional design used baseline data obtained through a site-specific ancillary protocol to a multisite trial evaluating the effectiveness of depression treatment (Sequenced Treatment Alternatives to Relieve Depression; STAR*D; http://www.star-d.org) that was designed to determine treatment resistance and test several medical and psychosocial treatments. ${ }^{27,28}$ After a baseline assessment, eligible participants were enrolled in Level 1, which consisted of up to 14 weeks of standardized treatment with a selective serotonin reuptake inhibitor (citalopram). Those who achieved remission with and could tolerate the Level 1 treatment then proceeded into a naturalistic follow-up phase, whereas those who did not could enter a series of randomized trials involving alternative antidepressants, psychotherapy, or both (Levels 2-5). Clinical Research Coordinators were trained and certified in implementing the screening procedures, inclusion and exclusion criteria, and data collection procedures. Research outcome data were independently collected by telephone interviews conducted by trained Research Outcome Assessors and by a telephone-based
Interactive Voice Response system. Data were collected in English or Spanish depending on patient preference. Both the overall STAR*D trial and this single-site ancillary study were approved by the University of Michigan Medical School Institutional Review Board. The main results of $\mathrm{STAR}^{*} \mathrm{D}$ are published elsewhere. ${ }^{29}$

\section{Participants}

Participants were a series of Michigan-site STAR*D trial enrollees recruited through advertisements and physician referral from either a psychiatric clinic or a family practice clinic, both affiliated with the University of Michigan Health System. Participants were required to be between 18 and 75 years of age and to currently meet criteria of the Diagnostic and Statistical Manual of Mental Disorders, Fourth Edition (DSM-IV) for a unipolar, nonpsychotic major depressive episode. Exclusion criteria included prior inadequate response to a robust trial of any of the protocol treatments, history of obvious intolerance to a protocol treatment during the current major depressive episode, current bipolar or eating disorder, history of psychosis, imminent need for medical or psychiatric hospitalization, and dementia.

\section{Procedures}

After giving informed consent but before filling the prescription for the antidepressant, eligible participants met with a Clinical Research Coordinator for baseline assessment of psychiatric status, psychiatric and medical history, demographics, and other study variables.

\section{Psychiatric Assessments}

We assessed the severity of depressive symptoms with the 17-item Hamilton Rating Scale for Depression (HAMD17). ${ }^{30}$ Comorbid psychiatric disorders were assessed with the Psychiatric Diagnostic Screening Questionnaire (PDSQ) ${ }^{31}$ which also covers DSM-IV criteria for dysthymia, generalized anxiety disorder, obsessive-compulsive disorder, panic disorder, agoraphobia, social phobia, posttraumatic stress disorder, somatoform disorder, hypochondriasis, alcohol abuse, substance abuse, and bulimia. The scale also assesses symptoms associated with suicidality, mania, and psychosis. Because the DSM-IV symptom duration requirement varies by disorder, the PDSQ incorporates 3 different time frames. The Cronbach $\alpha$ averages .86 across the 13 subscales, 1 -week test-retest stability is .83 , and considerable data support the measure's convergent and discriminant validity. ${ }^{31}$ Positive scores for each syndrome other than major depressive disorder were generated using subscale-specific cutoffs corresponding to $90 \%$ specificity. ${ }^{31,32}$ In addition, multiaxial psychiatric diagnoses were made by 2 licensed social workers who reviewed all study psychometric data and conducted a research interview based on DSM-IV criteria. 


\section{Comorbid Medical Conditions}

We assessed comorbid medical conditions with the Cumulative Illness Rating Scale (CIRS) ${ }^{33}$ administered by study personnel using a manual to gauge the severity of conditions within different organ systems. The CIRS is scored from 0 to 4 , with 0 indicating no problem $_{i} 1$, current mild problem or past marked problem; 2 , moderate disability or morbidity requiring therapeutic treatment; 3 , severe disability; and 4, very severe disability. The mean score for the severity-weighted item was computed for analysis, after excluding the psychiatric illness category. Interrater reliability for review of medical chart data is good (range, .78-.88), and the measure correlates well with patient-rated functional disability and physician-estimated disease burden. ${ }^{34}$

\section{Medication Beliefs}

We measured medication beliefs with the Beliefs about Medicines Questionnaire (BMQ), ${ }^{35}$ which assesses both medication-specific and general beliefs using 5-point Likert agreement-disagreement scales. The medication-specific scales - the necessity scale (a measure of perceived need) and the concerns scale (a measure of perceived harmfulness) - each consist of 5 items, such as, "My current mental health depends upon my antidepressant medication" (necessity) and "I sometimes worry about becoming too dependent upon my antidepressant" (concerns), for all items, higher scores indicate greater perceived need for medication or greater concerns about medication. The Cronbach $\alpha$ in the current study was .68 for both scales.

\section{Depression Representations}

We measured representations of depression using items from the Illness Perception Questionnaire-Revised (IPQ-R). ${ }^{36}$ Its subscales_-symptom identity, cause, timeline (acute vs chronic), consequences, control/cure (by self vs by formal treatment), cyclical timeline, and emotional representations - are internally consistent (Cronbach $\alpha \geq .79$ ) and show both discriminant and predictive validity. To abbreviate the 53 -item scale, we administered only the strongest-loading item from each factor subscale; however, we administered the Causes scale and added new items specific to depression, namely, stressful events, genetics, brain/chemical imbalance, germ/virus, chance/bad luck, personal relationships, physical reaction to medical illness, emotional reaction to medical illness, poor self-care, and up to 3 write-ins.

\section{Other Assessments}

We assessed patient characteristics with open-ended and multiple-choice items covering age, income, race, ethnicity, education, insurance status, living situation, recruitment site, and so forth. Additional depression characteristics were assessed though a combination of interview and self-report items concerning such issues as number of prior depressive episodes, duration of current episode, and related aspects, and medical chart reviews were used to supplement STAR*D data on prior antidepressant use.

\section{Data Analysis}

We analyzed the data using SPSS version 11.02 for Mac OS X (SPSS, Inc, Chicago, Illinois). Descriptive statistics were calculated to characterize the sample and evaluate central tendency, variability, and distribution for key variables. Continuous variables with a skewness index exceeding 0.4 were converted to ranks for data analysis. Missing data were not imputed. Zeroorder associations were evaluated using $\chi^{2}$ statistics for purely categorical associations, Student $t$ tests for categorical by continuous associations, and Pearson correlations for continuous associations.

We used linear multivariate regression to model perceptions about antidepressants as a function of demographics, depression features, and depression representations variables, applying the forward-selection entry procedure (entry $P<.05$; removal $P \geq .10$ ) to identify a parsimonious model. We fitted separate regression models to determine which components of the obtained model applied to the perceived necessity and harmfulness constructs individually. The forwardselection regression analyses were initially adjusted for age, sex, education, and site. The second blocks tested selected depression characteristics (number of prior episodes, years since first episode, prior antidepressant use, atypical depression, severity of medical comorbidity). The third and final blocks consisted of the depression representations variables: long future timeline, cyclical timeline, consequences, coherence (subjective sense of understanding the symptoms), and causes (stress, genes, virus, chemical imbalance, chance or bad luck, physical or emotional reaction to a medical condition, and poor self-care). We included a random effects term to adjust for correlated observations due to clustering of participants within clinical sites (primary care vs psychiatry).

\section{RESULTS}

\section{Descriptive Statistics}

Characteristics of the 165 study participants are shown in Table 1. In general, participants were young to middle-aged (mean \pm SD age, $34.2 \pm 10.5$ years) and had private insurance. Typically, they had moderate to severe depressive symptoms, 1 or 2 comorbid medical conditions, 1 comorbid Axis I disorder (most typically social phobia, present in $24.3 \%$ ), and 3 prior depressive 
episodes, but no prior antidepressant use. Scores for perceived necessity and perceived harmfulness of antidepressants were each centered around their midpoints (mean \pm SD, $3.04 \pm 0.48$ and $2.94 \pm 0.69$, respectively) and normally distributed (both skewness indices $\leq 0.27$ ), similar to what we have observed in clinical samples. ${ }^{14}$

\section{Bivariate Analysis}

Zero-order Pearson and Spearman correlations indicated that perceived necessity was significantly associated with age $(r=.27, P<.001)$, but not with sex, minority race or ethnicity, years of education, and health insurance status (all $P>$.34). Turning to clinical characteristics, perceived necessity was significantly associated with depressive symptom severity $(r=.31$, $P<.001)$, number of prior depressive episodes $(r=.26$, $P=.001)$, years since first episode $(r=.27, P<.001)$, prior antidepressant use $(r=.24, P=.003)$, and the presence of atypical depression $(r=.19, P=.02)$, but not with the presence of any specific comorbid Axis I or Axis II disorder, the presence of melancholic or anxious depression, duration of current episode, clinical

\begin{tabular}{|c|c|}
\hline Characteristic & Value \\
\hline Sex (female), \% & 57.5 \\
\hline Age, years, mean $\pm S D$ & $34.2 \pm 10.5$ \\
\hline \multicolumn{2}{|l|}{ Race, \% } \\
\hline White & 78.0 \\
\hline African American & 11.7 \\
\hline Asian & 2.6 \\
\hline American Indian or Alaskan Native & 1.0 \\
\hline Native Hawaiian or Pacific Islander & 1.6 \\
\hline Other or unspecified & 5.1 \\
\hline Ethnicity (Hispanic), \% & 4.6 \\
\hline Education, years, mean $\pm S D$ & $15.1 \pm 3.4$ \\
\hline Private health insurance, \% & 77 \\
\hline $\begin{array}{l}\text { Number of nonpsychiatric comorbid } \\
\text { conditions, mean } \pm \text { SD }\end{array}$ & $1.4 \pm 1.5$ \\
\hline Severity of illness, CIRS score, mean \pm SD & $1.07 \pm 0.50$ \\
\hline \multicolumn{2}{|l|}{ Depression characteristics } \\
\hline $\begin{array}{l}\text { Symptom severity (HAM-D17 total score), } \\
\text { mean } \pm \text { SD }\end{array}$ & $20.7 \pm 4.0$ \\
\hline $\begin{array}{l}\text { Duration of current episode, months, } \\
\text { median (IQR) }\end{array}$ & $16(8-34)$ \\
\hline Number of prior episodes, median (IQR) & $3(2-6)$ \\
\hline $\begin{array}{l}\text { Age of onset for first episode, years, } \\
\text { median (IQR) }\end{array}$ & $20(15-30)$ \\
\hline Axis I comorbidity, \% & 57.7 \\
\hline Prior antidepressant use, \% & 34.1 \\
\hline Depression in first-degree relative(s), \% & 51.1 \\
\hline \multicolumn{2}{|c|}{$\begin{array}{l}\text { CIRS = Cumulative Illness Rating Scale (possible scores: } 0-56 \text {, higher scores } \\
\text { indicate more severe illness); HAM-D17 = } 17 \text {-item Hamilton Rating Scale } \\
\text { for Depression (possible total scores: } 0-52 \text {, higher total scores indicate more } \\
\text { severe depression); IQR }=\text { interquartile range. }\end{array}$} \\
\hline
\end{tabular}

site (primary care or psychiatry), age of onset for first depressive episode, or history of depression in firstdegree relatives (all $P>.11$ ).

Perceived harmfulness was significantly associated with only 2 of the above variables: not having ever taken an antidepressant $(r=.18, P=.03)$ and low medical comorbidity burden $(r=-.16, P=.048)$.

Perceived necessity and harmfulness were not intercorrelated $(r=-.09, P=.21)$, replicating prior findings in depression $^{14,31}$ and other conditions, ${ }^{21}$ and suggesting that the constructs are orthogonal. With the exception of control covariates selected a priori, variables without a significant zero-order association with either perceived necessity or harmfulness were not examined in regression analyses.

\section{Multivariate Analysis}

As can be seen in Table 2, the results of multivariate analysis indicated that perceived necessity was not significantly related to any demographic variables except age $(P<.001)$, so that older patients reported greater perceived necessity. In terms of clinical variables, perceived necessity was higher among patients with more severe depressive symptoms $(P=.03)$ and a greater number of prior episodes, although this latter effect became nonsignificant when the third block added 2 depression representations variables to the final model $(P=.03$ and .09 , respectively). The valences of these latter effects indicated that perceived necessity was higher among patients who strongly endorsed the belief that their symptoms would have a long timeline $(P=.001)$ and who strongly attributed their symptoms to chemical imbalance $(P=.005)$. The total $R^{2}$ for perceived necessity was 30 .

Turning to perceived harmfulness, no demographic variables accounted for beliefs. Perceived harmfulness was however highest among patients who had no prior history of antidepressant use $(P=.02)$, believed that their symptoms were caused by bad luck or chance $(P=.04)$, and described themselves as having a poor understanding of their symptoms $(P=.003)$. The total $R^{2}$ for perceived harmfulness was .13 .

Neither perceived necessity nor perceived harmfulness was significantly associated with sex, level of education, whether the patient received mental health care at a primary care or psychiatry site, medical comorbidity, or any depression subtype (anxious, melancholic, and atypical).

\section{DISCUSSION}

Generalizing across conditions and medical regimens, Horne $^{37}$ has asserted that medication beliefs are "the hidden determinant of treatment outcome." At least 
in relation to antidepressants, beliefs appear to be a major-and in some studies the only_-predictor of adherence. They are clinically accessible, can be a valuable guide to physician decision making, and are potentially modifiable. A review by Demyttenaere ${ }^{38}$ concluded that antidepressant adherence improves when patient-clinician communication focuses on patients' personalized beliefs about how to best manage their depressive symptoms, which often conflict with medical models of treatment. Despite these assertions, very little research has attempted to explain what underlies this key variable.
Our current findings suggest that depressed patients' beliefs about antidepressants are associated with a rational and limited set of variables, as depicted in Figure 1. Perceived need for antidepressant medication was associated with older age, greater symptom severity, the perception that one's symptoms are caused by biochemical factors, and the expectation that one's symptoms will persist. These associations might guide clinicians who are aiming to improve depression outcomes. If nonadhering patients seem to underestimate the severity or persistence of their depressive symptoms, physicians might wish to highlight the historical impact of the

\begin{tabular}{|c|c|c|c|}
\hline $\begin{array}{l}\text { Dependent } \\
\text { Variable }\end{array}$ & Independent Variable & $\beta$ & $\begin{array}{c}P \\
\text { Value }^{a}\end{array}$ \\
\hline \multirow[t]{7}{*}{ Necessity belief ${ }^{b}$} & Age & .27 & $<.001$ \\
\hline & Sex & -.06 & .34 \\
\hline & Years of education & -.05 & .65 \\
\hline & $\begin{array}{l}\text { Depressive symptom severity (HAM-D17 total } \\
\text { score) }\end{array}$ & .17 & .03 \\
\hline & Number of prior episodes & .12 & $.09 c$ \\
\hline & Belief that symptoms will last a long time & .26 & .001 \\
\hline & $\begin{array}{l}\text { Belief that symptoms were caused by a } \\
\text { chemical imbalance }\end{array}$ & .22 & .005 \\
\hline \multirow[t]{6}{*}{ Harmfulness belief ${ }^{d}$} & Age & .00 & .99 \\
\hline & Sex & .00 & .99 \\
\hline & Years of education & .03 & .69 \\
\hline & History of antidepressant use & -.17 & .02 \\
\hline & Belief that symptoms were randomly caused & .21 & .04 \\
\hline & Subjective poor understanding of symptoms & .19 & .003 \\
\hline \multicolumn{4}{|c|}{ HAM-D17 = 17-item Hamilton Rating Scale for Depression. } \\
\hline \multicolumn{4}{|c|}{$\begin{array}{l}\text { All effects are adjusted for correlation due to clustering of patients within clinics. } \\
\text { b Total } R^{2}=.30 \text {. } \\
\text { ' Retained because this variable did not meet removal criterion of } P \geq .10 \text { in second block. } \\
\text { d Total } R^{2}=.13 \text {. }\end{array}$} \\
\hline
\end{tabular}

symptoms and discuss the typical chronicity of depression. It may be similarly beneficial to help patients develop realistic expectations about whether their symptoms will remit without treatment. Addressing these factors explicitly might enable patients to make a more fully informed choice about whether to use antidepressants. On the other hand, clinician indifference to this issue may increase the risks of nonadherence, undertreatment, and nonrecovery.

Importantly, perceived necessity was unrelated to several other indicators that have traditionally been associated with poor depression outcomes, including psychiatric comorbidity, age at first episode, and melancholic or anxious depression. This finding may seem surprising from a clini-

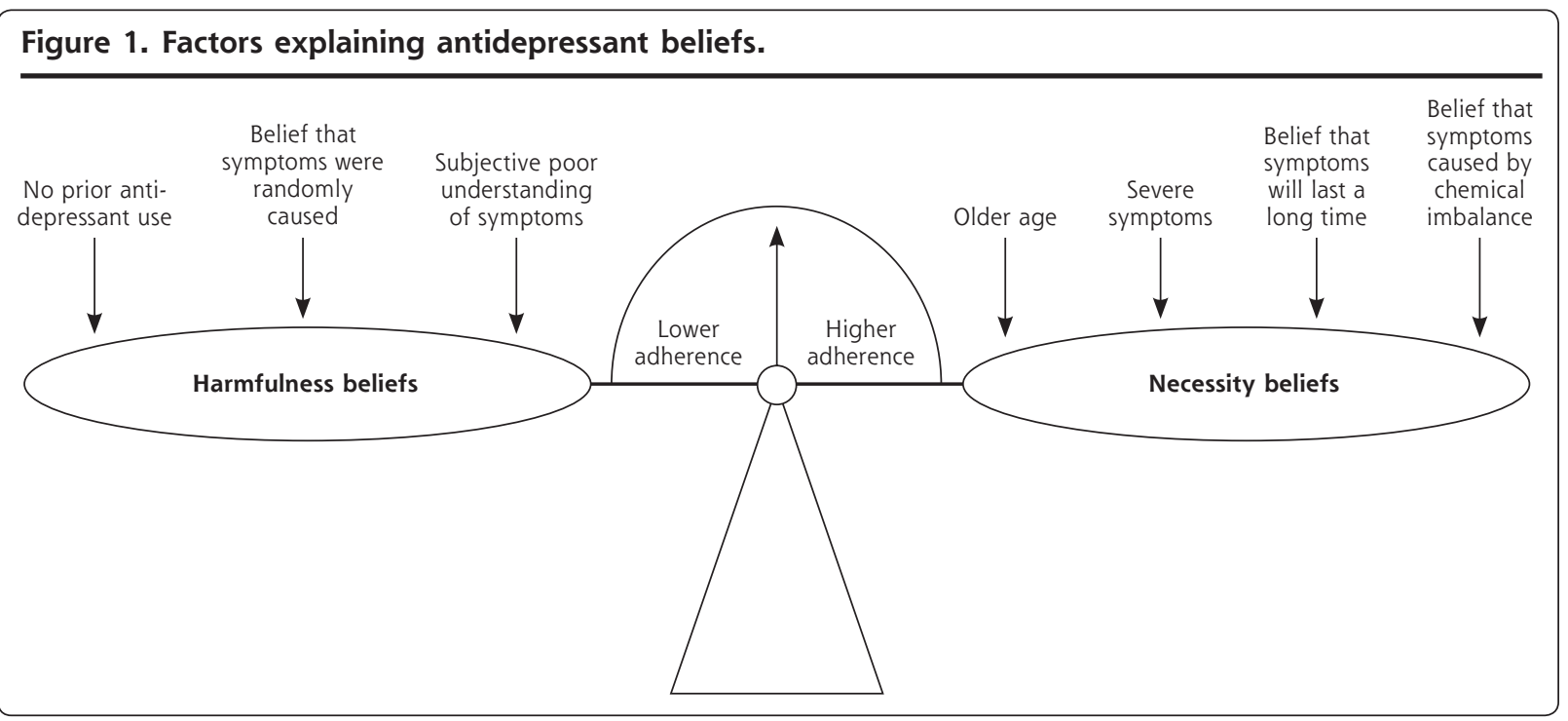


cal perspective, because each factor would be expected to enhance motivation for relief. Yet as we have argued elsewhere, ${ }^{39}$ symptom severity is probably much more distressing to affected individuals than the presence vs absence of a nosologically defined syndrome.

Perceived harmfulness was orthogonally related to perceived necessity and accordingly was explained by a completely different set of variables. Harmfulness beliefs were strongest among patients who had never taken antidepressants before and patients who lacked a clear notion of what was causing their symptoms. We speculate that, in lieu of personal experience with taking antidepressants, their primary information sources are media, culture, anecdotes, and past experience with nonpsychotropic medications. In this clinical situation, adherence might improve if clinicians carefully clarified the patient's specific concerns (eg, adverse effects, addiction, personality change, financial cost, stigma) and then offered treatment alternatives that respect these sensitivities. For example, this might translate to prescribing a conservative dosing and titration schedule that patients can self-pace and providing specific educational input where appropriate. Treating physicians might also encourage these patients to adopt an experimental waitand-see approach before drawing their conclusions.

The association between perceived harmfulness and random/chance causation suggests that harmfulness perceptions may be driven by the patients' lack of a clear cognitive model of depression. Evidently this is not attributable to a lack of experience in being depressed; in this study, depression history did not account for harmfulness beliefs (and random/chance causes continued to do so) even when treatment history was removed from consideration. Some of these patients may be actively disinterested in their own psychology, may prefer a more directive physician style, or both. Others may be receptive to developing a more subjectively clear, personalized model of distress. These individuals might benefit from openended exploration about what they believe might help them and possibly from exposure to a menu of other models of depression. Alternatively, a patient's belief in random/chance causes may reflect a general rejection of psychological determinism. Such patients ought to likewise be allowed to openly discuss their views about depression and encouraged to adopt a blend of treatment strategies that they find acceptable.

Study limitations include the self-report methodology necessitated by the subject matter and sample size. Internal validity could be improved in future studies by using multiple data sources, such as noteworthy reports and archival data. Self-selection and referral bias probably led to overrepresentation of patients with positive attitudes toward medication, and therefore the find- ings may not apply to all patients entering depression treatment. This problem may have been compounded by the exclusion of potential participants who previously had either a prior inadequate response or severe adverse effects from protocol treatments. Our descriptive data on beliefs, however, indicate that the sample's beliefs scores were typical of those seen in clinical samples, centered about the midpoint, and normally distributed. The total variance explained was somewhat low $\left(R^{2}=.13\right)$ in the case of harmfulness beliefs. We assessed beliefs after patients were prescribed an antidepressant (but before they left the clinic to fill the prescription). This approach raises the possibility that clinic physicians may have influenced patients' beliefs; however, if we had assessed beliefs before the medical encounter, we would not have fully captured usual care conditions. Future research might aim to confirm the findings in a more clinically representative sample of all treatment-eligible patients.

To summarize, the present findings imply that patients' beliefs about the necessity and safety of antidepressants are independent of each other and related to different sets of factors. Belief-focused interventions to enhance adherence may be maximally effective if oriented toward patients who (1) underestimate their symptom severity, (2) believe that their symptoms will be temporary despite past experiences to the contrary, (3) have not previously taken antidepressants, (4) believe that their symptoms are randomly caused, or (5) feel subjectively bewildered by their symptoms.

Although patients' beliefs about depression and its treatment might be a useful guide to clinical decision making, clinicians should bear in mind that negative attitudes about treatment may be either accurately or inaccurately based on prior experiences, and thus it might not always be appropriate to attempt to modify patients' beliefs that are interfering with adherence. Although our current data do not directly address this point, in an earlier study we found that negative attitudes about treatment predict good 9-month outcomes, even after adjusting for depression severity and history. ${ }^{11}$ Given the limitations of the current cross-sectional data, we are now following the present patient sample throughout the entire treatment course. The resulting data will help reveal how these key beliefs evolve during treatment and indicate which variables should be targeted to enhance adherence and outcome.

To read or post commentaries in response to this article, see it online at http://www.annfammed.org/cgi/content/full/6/1/23.

Key words: Depressive disorder; antidepressive agents; patient compliance; patient nonadherence; beliefs; patient-centered care

Submitted May 10, 2007; submitted, revised, July 30, 2007; accepted August 8, 2007. 
Funding support: The parent project (STAR*D) was supported by funding from the National Institute of Mental Health, National Institutes of Health, under contract N01MH90003.

Disclaimer: The content of this publication does not necessarily reflect the views or policies of the Department of Health and Human Services, nor does mention of trade names, commercial products, or organizations imply endorsement by the US Government.

Acknowledgments: We are grateful for the superb assistance we received from Hedieh Briggs, MSW, LCSW, and Kate Harris Bullard, MPH, LSCW, Clinical Research Coordinators at the University of Michigan. We also thank Stephen R. Wisniewski, PhD, and Robert Gatt from the STAR*D Data Coordinating Center at University of Pittsburgh.

\section{References}

1. Depression Guideline Panel. Depression in Primary Care: Volume 1. Detection and Diagnosis. Clinical Practice Guideline, Number 5. Rockville, MD: US Department of Health and Human Services, Public Health Service, Agency for Health Care Policy and Research; 1993. AHCPR publication 93-0550.

2. Maixner SM, Greden JF. Extended antidepressant maintenance and discontinuation syndromes. Depress Anxiety. 1998;8(Suppl 1):43-53.

3. American Psychiatric Association. Practice guideline for the treatment of patients with major depressive disorder. Am J Psychiatry. 2000;157(4 Suppl):1-45.

4. Peveler R, Grorge C, Kinmonth A, Campbell M, Thompson C. Effect of antidepressant drug counselling and information leaflets on adherence to drug treatment in primary care: randomized controlled trial. BMJ. 1999;319(7210):612-615.

5. Lin $E$, Von Korff $M$, Katon W, et al. The role of the primary care physician in patients' therapy. Med Care. 1995;33(1):67-74.

6. Bultman DC, Svarstad BL. Effects of pharmacist monitoring on patient satisfaction with antidepressant medication therapy. J Am Pharm Assoc. 2002;42(1):36-43.

7. Melfi CA, Chawla AJ, Croghan TW, et al. The effects of adherence to antidepressant treatment guidelines on relapse and recurrence of depression. Arch Gen Psychiatry. 1998;55(12):1128-1132.

8. Frank E, Perel JM, Mallinger AG, et al. Relationship of pharmacologic compliance to long-term prophylaxis in recurrent depression. Psychopharmacol Bull. 1992;28(3):231-235.

9. Sood N, Treglia M, Obenchain RL, et al. Determinants of antidepressant treatment outcome. Am J Manag Care. 2000;6(12):1327-1336.

10. DiMatteo MR, Haskard KB, Williams SL. Health beliefs, disease severity, and patient adherence: a meta-analysis. Med Care. 2007;45(6):521-528.

11. Aikens JE, Kroenke K, Swindle RW, Eckert GJ. Nine-month predictors and outcomes of SSRI antidepressant continuation in primary care. Gen Hosp Psychiatry. 2005;27(4):229-236.

12. Schwenk TL, Evans DL, Laden SK, Lewis L. Treatment outcome and physician-patient communication in primary care patients with chronic recurrent depression. Am J Psychiatry. 2004;161(10):1892-1901.

13. Givens JL, Datto CJ, Ruckdeschel K, et al. Older patients' aversion to antidepressants: a qualitative study. J Gen Intern Med. 2006;21(2) 146-151.

14. Aikens JE, Nease DE Jr, Nau DP, Klinkman MS, Schwenk TL. Adherence to maintenance-phase antidepressant medication as a function of patient beliefs about medication. Ann Fam Med. 2005;3(1):23-30.

15. Kutcher S, Leblanc J, Maclaren C, Hadrava V. A randomized trial of a specific adherence enhancement program in sertraline-treated adults with major depressive disorder in a primary care setting. Prog Neuropsychopharmacol Biol Psychiatry. 2002;26(3):591-596.

16. Brook OH, van Hout $\mathrm{H}$, Stalman W, et al. A pharmacy-based coaching program to improve adherence to antidepressant treatment among primary care patients. Psychiatr Serv. 2005;56(4):487-489.
17. Pampallona S, Bollini P, Tibaldi G, et al. Combined pharmacotherapy and psychological treatment for depression: a systematic review. Arch Gen Psychiatry. 2004;61(7):714-719.

18. Pampallona S, Bollini P, Tibaldi G, et al. Patient adherence in the treatment of depression. Br J Psychiatry. 2002;180:104-109.

19. Katon W, Rutter C, Ludman EJ, et al. A randomized trial of relapse prevention of depression in primary care. Arch Gen Psychiatry. 2001;58(3):241-247.

20. Petrie KJ, Jago LA, Devcich DA. The role of illness perceptions in patients with medical conditions. Curr Opin Psychiatry. 2007;20(2): 163-167.

21. Horne R, Weinman J. Patients' beliefs about prescribed medicines and their role in adherence to treatment in chronic physical illness. J Psychosom Res. 1999;47(6):555-567.

22. Clifford S, Barber N, Elliott R, Hartley E, Horne R. Patient-centered advice is effective in improving adherence to medicines. Pharm World Sci. 2006;28(3):165-170.

23. Brown C, Battista DR, Bruehlman R, et al. Beliefs about antidepressant medications in primary care patients: relationship to selfreported adherence. Med Care. 2005;43(12):1203-1207.

24. Leventhal $H$, Diefenbach $M$, Leventhal EA. Illness cognition: using common sense to understand treatment adherence and affect cognition treatment. Cognit Ther Res. 1992;16(2):143-163.

25. Brown C, Dunbar-Jacob J, Palenchar DR, et al. Primary care patients' personal illness models for depression: a preliminary investigation. Fam Pract. 2001;18(3):314-320.

26. Sullivan MD, Katon WJ, Russo JE, et al. Patient beliefs predict response to paroxetine among primary care patients with dysthymia and minor depression. J Am Board Fam Pract. 2003;16(1):22-31.

27. Fava M, Rush AJ, Trivedi MH, et al. Background and rationale for the Sequenced Treatment Alternatives to Relieve Depression (STAR*D) study. Psychiatr Clin North Am. 2003;26(2):457-494.

28. Rush AJ, Fava M, Wisniewski SR, et al. Sequenced Treatment Alternatives to Relieve Depression (STAR*D): rationale and design. Control Clin Trials. 2004;25(1):119-142.

29. Rush AJ, Trivedi MH, Wisniewski SR, et al. Bupropion-SR, sertraline, or venlafaxine-XR after failure of SSRIs for depression. N Engl J Med. 2006;354(12):1231-1242.

30. Hamilton MJ. A rating scale for depression. J Neurol Neurosurg Psychiatry. 1960;23:56-62.

31. Zimmerman M, Mattia JI. A self-report scale to help make psychiatric diagnoses: the Psychiatric Diagnostic Screening Questionnaire. Arch Gen Psychiatry. 2001;58(8):787-794.

32. Rush AJ, Zimmerman M, Wisniewski SR, et al. Comorbid psychiatric disorders in depressed outpatients: demographic and clinical features. J Affect Disord. 2005;87(1):43-55.

33. Linn BS, Linn MW, Gurel L. Cumulative illness rating scale. J Am Geriatr Soc. 1968;16(5):622-626.

34. de Groot V, Beckerman H, Lankhorst GJ, Bouter LM. How to measure comorbidity. A critical review of available methods. J Clin Epidemiol. 2003;56(3):221-229.

35. Horne R, Weinman J, Hankins M. The Beliefs about Medicines Questionnaire (BMQ): the development and evaluation of a new method for assessing the cognitive representation of medication. Psychol Health. 1998;14:1-24.

36. Moss-Morris R, Weinman J, Petrie KJ, et al. The Revised Illness Perception Questionnaire (IPQ-R). Psychol Health. 2002;17(1):1-16.

37. Horne R. Patients' beliefs about treatment: the hidden determinant of treatment outcome? J Psychosom Res. 1999;47(6):491-495.

38. Demyttenaere K. Risk factors and predictors of compliance in depression. Eur Neuropsychopharmacol. 2003;13(Suppl 3):S69-S75.

39. Nease DE Jr, Aikens JE. DSM depression and anxiety criteria and severity of symptoms in primary care: cross sectional study. BMJ. 2003;327(7422):1030-1031. 